\title{
Radar Sounding Using the Cassini Altimeter: Waveform Modeling and Monte Carlo Approach for Data Inversion of Observations of Titan's Seas
}

\author{
Marco Mastrogiuseppe, A. Hayes, V. Poggiali, R. Seu, Jonathan I. Lunine, and J. D. Hofgartner
}

\begin{abstract}
Recently, the Cassini RADAR has been used as a sounder to probe the depth and constrain the composition of hydrocarbon seas on Saturn's largest moon, Titan. Altimetry waveforms from observations over the seas are generally composed of two main reflections: the first from the surface of the liquid and the second from the seafloor. The time interval between these two peaks is a measure of sea depth, and the attenuation from the propagation through the liquid is a measure of the dielectric properties, which is a sensitive property of liquid composition. Radar measurements are affected by uncertainties that can include saturation effects, possible receiver distortion, and processing artifacts, in addition to thermal noise and speckle. To rigorously treat these problems, we simulate the $\mathrm{Ku}$-band altimetry echo received from Titan's seas using a two-layer model, where the surface is represented by a specular reflection and the seafloor is modeled using a facet-based synthetic surface. The simulation accounts for the thermal noise, speckle, analog-to-digital conversion, and block adaptive quantization and allows for possible receiver saturation. We use a Monte Carlo method to compare simulated and observed waveforms and retrieve the probability distributions of depth, surface/subsurface intensity ratio, and subsurface roughness for the individual double-peaked waveform of Ligeia Mare acquired by the Cassini spacecraft in May 2013. This new analysis provides an update to the Ku-band attenuation and results in a new estimate for its loss tangent and composition. We also demonstrate the ability to retrieve bathymetric information from saturated altimetry echoes acquired over Ontario Lacus in December 2008.
\end{abstract}

Index Terms-Altimetry, dielectric constant, geophysical measurement techniques, geophysical signal processing, ground penetrating radar, radar, spaceborne radar, Titan.

\section{INTRODUCTION}

$\mathbf{R}$ ADAR sounders are a valuable tool for planetary exploration and have been used to probe the subsurface of

Manuscript received April 10, 2016; accepted April 18, 2016. Date of publication July 11, 2016; date of current version August 11, 2016. This work was supported in part by the National Aeronautics and Space Administration under Grants NNX13AG03G, NNX15AH10G, and NNX14AJ57G and in part by the Italian Space Agency (ASI) through Contract 2014-041-R.0 J.D.H. gratefully acknowledges support from the Natural Sciences and Engineering Research Council and the Cassini project.

M. Mastrogiuseppe is with the Cornell Center for Astrophysical Science, Cornell University, Ithaca, 14850 NY USA, and also with the Dipartmento di Ingegneria dell'Informazione, Elettronica e Telecomunicazioni (DIET), Sapienza Universita' di Roma, 00185 Roma, Italy (e-mail: mastrogiuseppe@ uniroma1.it).

A. Hayes, J. I. Lunine, and J. D. Hofgartner are with the Cornell Center for Astrophysical Science, Cornell University, Ithaca, 14850 NY USA.

V. Poggiali and R. Seu are with the Dipartmento di Ingegneria dell'Informazione, Elettronica e Telecomunicazioni (DIET), Sapienza Universita' di Roma, 00185 Roma, Italy.

Color versions of one or more of the figures in this paper are available online at http://ieeexplore.ieee.org.

Digital Object Identifier 10.1109/TGRS.2016.2563426 planets and moons. Such instruments are generally designed for working at long wavelength in order to achieve large penetration depths. Some examples are MARSIS [1] and SHARAD [2] which are currently operating on Mars and have been able to detect subsurface structures at depths of up to a few kilometers under the surface. Another example is given by the Lunar Radar Sounder on board the Japanese Kaguya mission [3], which operated in orbit during 2008 and 2009 and revealed subsurface structures on the Moon. Airborne radar sounders were also used for terrestrial application; an example is the High-Capability Radar Sounder, which successfully collected a large amount of data during campaigns in Antarctica [4]. Future radar sounders include REASON [5] and RIME [6] that will study the subsurfaces of the ice-crusted Galilean moons and search for putative subsurface oceans and pockets of liquid water.

Herein, we present data from RADAR onboard the Cassini spacecraft and demonstrate the instrument's ability to act as a radar sounder that can probe seas of liquid hydrocarbon in Titan's polar terrain. Titan, the largest moon of Saturn, is the only body in the solar system, aside from Earth, known to have an active hydrologic cycle akin to our own water cycle. Titan's methane-based hydrologic system drives climatic processes that result in geomorphologic features, including dunes, fluvial channels, and large deposits of liquid hydrocarbons in lakes and seas that are strikingly similar to terrestrial counterparts [7].

While the microwave transparency of liquid hydrocarbons had been recognized prior to the launch of the Cassini-Huygens mission to Saturn [8], [9], the Cassini RADAR was not designed to be a radar sounder (e.g., having a high operating frequency of $13.8 \mathrm{GHz}$ ), and radar sounding was not a science objective. Recently, however, Mastrogiuseppe et al. [10] have shown that, despite a suboptimal frequency for sounding, altimetry observations of Titan's large sea, Ligeia Mare, exhibit returns from both the sea surface and sea floor (see Fig. 1). The sea depth was determined from the temporal distance between the two main peaks in the waveform (radar echo time series), and the speed of light in the liquid. The measured bathymetry profile had a maximum depth of $160 \mathrm{~m}$, suggesting a large sea volume that includes more hydrocarbons than all of the known terrestrial fossil fuel reserves [11], [43]. The loss tangent of the sea is determined by estimating the attenuation of the radar energy from the amplitude ratio of the two reflections received as a function of depth. The average loss tangent of Ligeia Mare was measured to be $3+/-1 \times 10^{-5}$, consistent with a ternary mixture of methane-ethane-nitrogen with little to no impurities. 


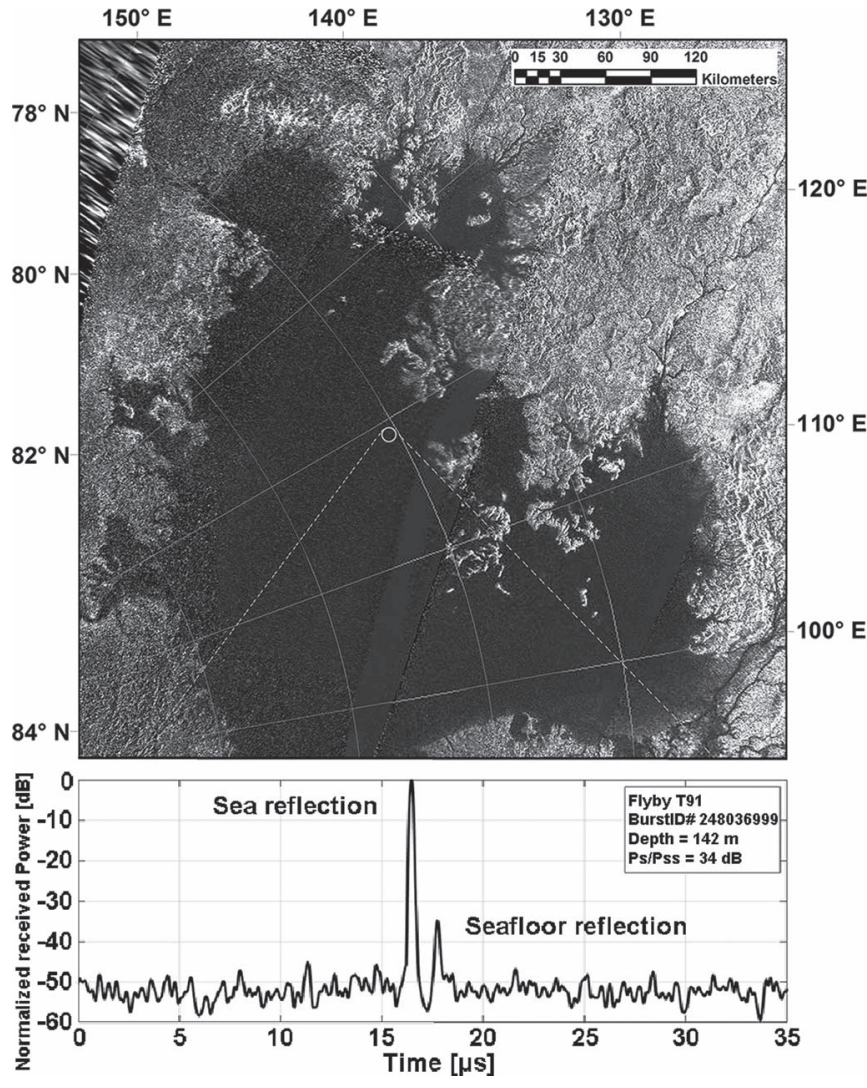

Fig. 1. Cassini synthetic aperture radar image of Titan's hydrocarbon sea, Ligeia Mare, and altimetry waveform from the T91 Cassini flyby that shows reflections from the sea surface and sea floor.

The concept of using multipeak intensity ratios as a function of depth to determine dielectric properties has been adopted previously [12], [13]. Zhang [14] used the technique to invert MARSIS data acquired at the South Pole of Mars. Similarly, the authors of [15]-[17] used the amplitude ratio of SHARAD data to obtain the loss tangent of the North polar layer deposits of Mars.

In all these examples, as well as the present case of Cassini data over Titan's seas, however, the inversion of radar sounding data can be complicated by the complexity of the observation and deficiencies in the model. For example, surface roughness generates a clutter signal that is superimposed on the subsurface signal of interest, and the heterogeneity of the sounded medium (e.g., stratification) can generate multiple returns that affect the amplitude of the subsurface returns. Furthermore, roughness and dielectric properties at the surface and at the subsurface interface can vary both spatially and temporally.

In the case of Cassini radar observations of Titan's seas and lakes, the inversion is simplified by the nature of the environment. The high power of the surface return has been used to demonstrate that the liquid surface is very smooth [18], [19], [45] at the 2.2-cm Cassini radar wavelength and thus does not introduce a clutter ambiguity. In addition, the liquid is remarkably transparent and shows no evidence for volume scattering (either from multilayer stratification or from suspended scatters), and thus, it can be assumed to be homogeneous [10]. Moreover, within the sea, the dielectric properties of the liquid are expected to be spatially invariable. All of these factors simplify the inversion problem.

Despite these simplifications, however, radar sounding using the Cassini RADAR system is challenging because it was never designed to operate as a sounder. For example, when the depths of the sea or lake approach the radar range resolution ( $\sim 35 \mathrm{~m}$ ), the two main lobes generated by the reflections at the interfaces interact with each other, complicating the measurement of the reflection amplitudes and time interval. Other problems include saturation effects that have occurred during the observation of liquid bodies such as Ontario Lacus. In the case of saturated bursts, the radar signal waveform is distorted, and the accurate identification of surface and subsurface peaks is hindered. Using such derived data products thus complicates inversion through the introduction of distortion, processing artifacts, thermal noise, speckle, etc. Such uncertainties are difficult to evaluate and can compromise the results of data inversion.

Herein, we treat radar measurements as stochastic variables described by a posterior probability density function that describes the uncertainties of the measured data used for inversion. Furthermore, our analysis simulates the raw Cassini radar altimeter waveform, incorporating all the operations that are applied by the onboard system and subsequent ground processing. This method allows the simulation to reproduce processing artifacts, including saturation effects introduced by the analogto-digital conversion (ADC) and block adaptive quantization (BAQ) systems [20]. Saturation artifacts, which can manifest as erroneous pre- and postcursor signal peaks after processing, are caused by the clip of raw signal amplitude, as also observed in terrestrial radar systems [21].

We use an in-flight Cassini RADAR engineering test conducted in June 2009 (T56) to characterize saturation effects and reproduce receiver distortion. The waveform received from liquid body observations is simulated using a two-layer model which accounts for seafloor roughness using a facet method. The best fit model coefficients and their confidence intervals are determined using a random walk Monte Carlo method coupled to a least square minimization between the time series (waveform) of the observed and simulated data that is guided by the prior probability density functions. A similar model-based approach is presented by Tarantola [22] as a possible technique to invert GPR and seismic data on Earth. However, due to the ill-posed nature of the inverse problem, our method aims only at evaluating the liquid's loss tangent by assuming a spatially constant permittivity over the region of interest.

We then use the derived best fit parameters as inputs to a parametric model that relates the loss tangent to liquid absorption and ultimately allows us to retrieve the composition assuming that the liquid represents a ternary nitrogenethane-methane mixture. As compared to the initial retrieval techniques described in [10], the method described herein permits a more complete characterization of the measurement errors, improves the derived spatial resolution, and presents a more robust determination of liquid depth and attenuation.

In Section II, we present the Cassini RADAR observation of Titan's large liquid bodies. In Section III, we present the 
Cassini radar simulation that is used to reproduce the observed waveform of Titan's seas and lakes. In Section IV, we present a method to estimate the most probable values and relative uncertainties of depths, intensity ratio, and vertical roughness using a Monte Carlo approach along with a waveform fitting of observed and simulated data, with specific case studies of saturated bursts over Ontario Lacus and unsaturated bursts over Ligeia Mare. In Section V, we use the previous estimates obtained in Section IV to update the loss tangent of Ligeia Mare. Finally, we discuss the implications of the new estimation for the loss tangent and suggest a possible composition of Ligeia Mare.

\section{CASSini RADAR Altimetry ObServation OF TITAN'S LIQUID BODIES}

The first Cassini RADAR image, which confirmed the presence of lakes on Titan, was acquired during the flyby T16 in July 2006 [23]. Later observations revealed the existence of other liquid bodies, including the major three seas Kraken Mare, Ligeia Mare, and Punga Mare located in the North Polar region and the largest lake Ontario Lacus in the South Polar region [24]-[26].

A successive altimetric observation of the second largest sea on Titan, Ligeia Mare, during the May 2013 (T91) flyby permitted the first direct measurement of its bathymetry and the relative estimation of its loss tangent [10]. As a result, altimetry observations of Kraken Mare and Punga Mare were planned and executed during the August 2014 (T104) and January 2015 (T108) flybys.

In altimeter mode, the 2.2-cm Cassini RADAR collects data by transmitting bursts of 21 chirps with a pulse repetition frequency of $5 \mathrm{kHz}$ and a signal bandwidth of $4.25 \mathrm{MHz}$, allowing a range resolution equal to $35 \mathrm{~m}$ in vacuum [27]. The burst repetition interval (BRI) can be adjusted, for example, in the case of T91 observation, the altimeter mode had a BRI of $3 \mathrm{~s}$. The receiver opens a time window that captures 15 of the 21 transmitted signals, and the data are sampled at $10 \mathrm{MHz}$. For each flyby, the digitized voltage returns are sent to Earth through the Deep Space Network and processed on the ground.

During the RADAR altimeter observation of Ligeia Mare and other liquid bodies, the Cassini altitude varied from 1500 to $2000 \mathrm{~km}$, leading to an along-track resolution of about $10 \mathrm{~km}$ which eventually can be improved using range Doppler processing [28]. Due to the geometry and system parameters, at these altitudes, Cassini RADAR works in beam-limited mode, and when the surface or subsurface roughness is moderate (i.e., rms heights smaller than the range resolution), the received compressed radar waveform has a Gaussian shape.

Observed waveforms acquired over Ligeia Mare show a narrow high peak intensity, indicative of the specular nature of the surface reflection [18], followed by a secondary weaker echo reflected from the sea floor [10]. When the time delay of the two reflections is large enough to allow the clear separation of the two signals (i.e., when depth is larger than the radar range resolution), the waveform shows no evidence for volume scattering (i.e., the signal level between surface and subsurface peaks reaches the noise level) (see, for example, Fig. 1).

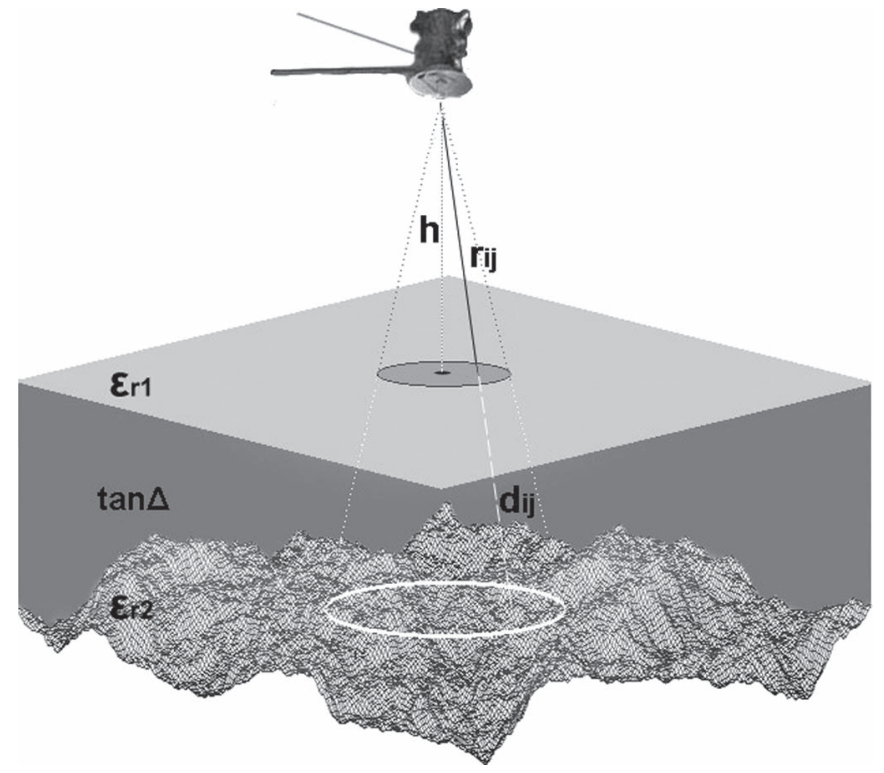

Fig. 2. Two-layer model of Cassini altimetry observation of liquid bodies (not to scale). The first reflection from the liquid surface is represented by a specular reflection, and the second reflection from the subsurface is represented as the sum of facet-based reflections.

In order to simulate this scenario, we modeled the received waveform by means of a two-layer model with smooth air-liquid and rough liquid-seafloor interfaces (see Fig. 2).

\section{Simulation of Cassini Altimetry WaVeforms FROM LIQUID BODIES ON TITAN}

We simulate Cassini RADAR altimetry waveforms of Titan's large liquid bodies using a two-layer model where the first interface (i.e., gas/liquid) is flat [18], [19] and represented by a single scatter located at the subsatellite point with a radar cross section described in [29]. The second interface (i.e., liquid/solid) is computed using a facet method as described in [30] and [31] or in [32] for simulating Titan's dunes (see Fig. 2).

We neglect multiple reflections between the two layers as well as volume scattering within the liquid. The subsurface is simulated according to the macroscale roughness parameters and the spectral function using $64 \times 64$ facets over a square area of $164 \mathrm{~km}^{2}$ (see Fig. 2). For the spectral function, we allow the macroscale rms height to vary but set the Hurst exponent (which is related to the fractal dimension) equal to 0.5 , which corresponds to the Brownian surface [33]. Each scenario is simulated in order to have a physical size larger than the $-3-\mathrm{dB}$ antenna pattern footprint. The scattering function at the scale of individual facets $(\sim 100 \mathrm{~m})$ is calculated using a Hagfors model [34] with a constant rms slope of $15^{\circ}$. In practice, this smallscale roughness parameter acts to change the absolute received power but does not affect the pulse shape, which is dominated by the facet-scale roughness. The small-scale roughness does not affect depth and/or loss tangent estimations as they are a function of time delay and intensity ratios, not absolute received power. As a result, the choice of $15^{\circ}$ is representative of Titan surfaces at the scale of wavelength [35], but arbitrary to the analysis described herein. 
From the waveform simulation of the Cassini beam-limited mode observations, we found that pulse shape is mainly dependent on the rms height rather than the autocorrelation function used to describe the surface slope distribution. We thus set the subsurface intensity Pss with respect to the surface intensity $P s$ at the desired intensity ratio on a scenario having a specular reflection at the surface and subsurface where only Hagfors scattering occurs. Then, we allow the subsurface vertical roughness to vary in order to simulate the shape of the observed echoes.

As a result, our model has three free parameters: depth, intensity ratio, and rms height of macroscale subsurface roughness. The simulated radar return is calculated as a superposition of the returns from each facet, properly shifted in time and scaled in amplitude with the Hagfors model and the antenna gain pattern, taking the incidence angle into account at each facet. The phase is also taken into account using the distance from the facets to the spacecraft.

The scattering signal (in volts) as a function of time from such scenario can be represented as

$$
\begin{aligned}
g(t)=a \delta(t & \left.-t_{0}\right) \exp (j 2 k h) \\
& +\sum_{i}^{N} \sum_{j}^{M} b_{i j} \delta\left(t-t_{i j}\right) \exp \left(j 2 k\left[r_{i j}+z_{i j}\right]\right)
\end{aligned}
$$

with $t_{0}=2 h / c, t_{i j}=2\left(r_{i j}+z_{i j}\right) / c$.

Where $h$ is the spacecraft altitude, $k$ is the wavenumber, $c$ is the speed of light, $\mathrm{N}$ and $\mathrm{M}$ are the number of facets for each dimension of the simulated subsurface, $r_{i j}$ is the distance from the spacecraft to the surface along the direction of the facets, $z_{i j}=d_{i j} / \sqrt{\varepsilon_{r 1}}$ accounts for the apparent additional path within the liquid, $\delta$ is the Dirac function, $\varepsilon_{\mathrm{r} 1}$ and $\varepsilon_{\mathrm{r} 2}$ are the real parts of the dielectric constants of the surface and subsurface, respectively, and $\tan \Delta$ is the loss tangent of liquid (see Fig. 2). The surface amplitude value $a$ is simulated according to the physical-optics model, while the value $b$ is set to give the desired Ps/Pss and it takes into account the attenuation, subsurface roughness, and dielectric properties of the two interfaces.

The received signal $s(t)$ is obtained by convolving $(\otimes)$ the modeled scattering response $g(t)$ with the transmitted signal $m(t)$

$$
s(t)=g(t) \otimes m(t) .
$$

The transmitted signal $m(t)$ is a chirp with a quadratic phase function that is generated according to the input parameters of the Cassini subsystem to the digital chirp generator: start frequency, stop frequency, chirp length, and step frequency [27]. The parameters are used to generate each of the 15 chirps contained within a received burst. For each pulse of the burst, the phase is calculated according to the movement of the spacecraft, allowing the simulation of speckle noise as well as the pulse-to-pulse correlation present in the observed waveforms.

\section{A. Noise Model}

We add white Gaussian noise to our simulated waveform. The noise amplitude is empirically determined using the interpulse noise measured between echoes of the T91 Ligeia observation. In case of saturated data, it is scaled according to the attenuator setting level and the relationship found in [36].

\section{B. $A D C$ and $B A Q$}

The 8-b analog-digital converter(ADC) of the CassiniRADAR discretizes the signal amplitudes between -5 and $+5 \mathrm{~V}$ into 255 levels ranging from -127.5 to $+127.5 \mathrm{dn}$ ( $\mathrm{dn}$ is data number). We reproduce signal saturation as a hard clip when the amplitude exceeds $\pm 127.5 \mathrm{dn}$. The Cassini 4-b BAQ compression algorithm is similar to the one adopted for the Magellan RADAR. We reproduced it by implementing the block schemes outlined in [20] and threshold calculations in [36].

\section{Distortion and Saturation: T56 Engineering Test}

We used the in-flight engineering test conducted during the June 2009 Titan flyby (T56) where ten bursts were routed directly from the transmitter to the receiver while sequentially incrementing the attenuation at steps of $2 \mathrm{~dB}$ in order to cause different levels of signal saturation. In order to characterize and reproduce distortion introduced by saturation, we low-pass filtered the received burst by means of a moving window of $20 \mu \mathrm{s}$. We noted that the saturation at the receiver introduces a time-varying dc offset of the received signal which is a function of the amplitude level input to the ADC. We represented this behavior by adding to each individual signal of the simulated burst, before ADC and BAQ, a linear time-varying function that decreases the slope with time as a function of the saturation level. We found a maximum slope of 2.3 digitized number $(\mathrm{dn}) / \mu$ s for an input amplitude of $570 \mathrm{dn}$.

The minimum value of the dc offset decreases monotonically from about 0 to $-45 \mathrm{dn}$ for a range of ADC input amplitudes from $90 \mathrm{dn}$ (when saturation occurs at $-60 \mathrm{~dB}$ ) to $450 \mathrm{dn}$ ( $-48 \mathrm{~dB}$ in Fig. 3), respectively, and, after that, tends to be constant up to $570 \mathrm{dn}(-45 \mathrm{~dB})$.

The simulated results are reported in Fig. 3 (right side) and are comparable to the observed distortion of the T56 engineering test (see left side of Fig. 3).

\section{Ground Processing and Simulated Waveform}

Simulated bursts are range compressed and incoherently averaged, as implemented in the Cassini Processing of Altimetric Data (CPAD) pipeline [37]. Before averaging, a superresolution algorithm is applied, as described in the supplementary information in [10]. The result is a time-sampled waveform which represents the response of the radar to the simulated scenario. The simulated waveforms are able to accurately reproduce the actual observations, as shown in Fig. 5 where unsaturated echoes from Ligeia Mare and saturated echoes from Ontario Lacus observations are both shown.

\section{Monte Carlo Simulation AND WAVEFORM APPROACH}

The inversion method adopted in this is carried out in two separate steps. The first step determines the most probable values and relative errors of the best fit depth, intensity ratio 

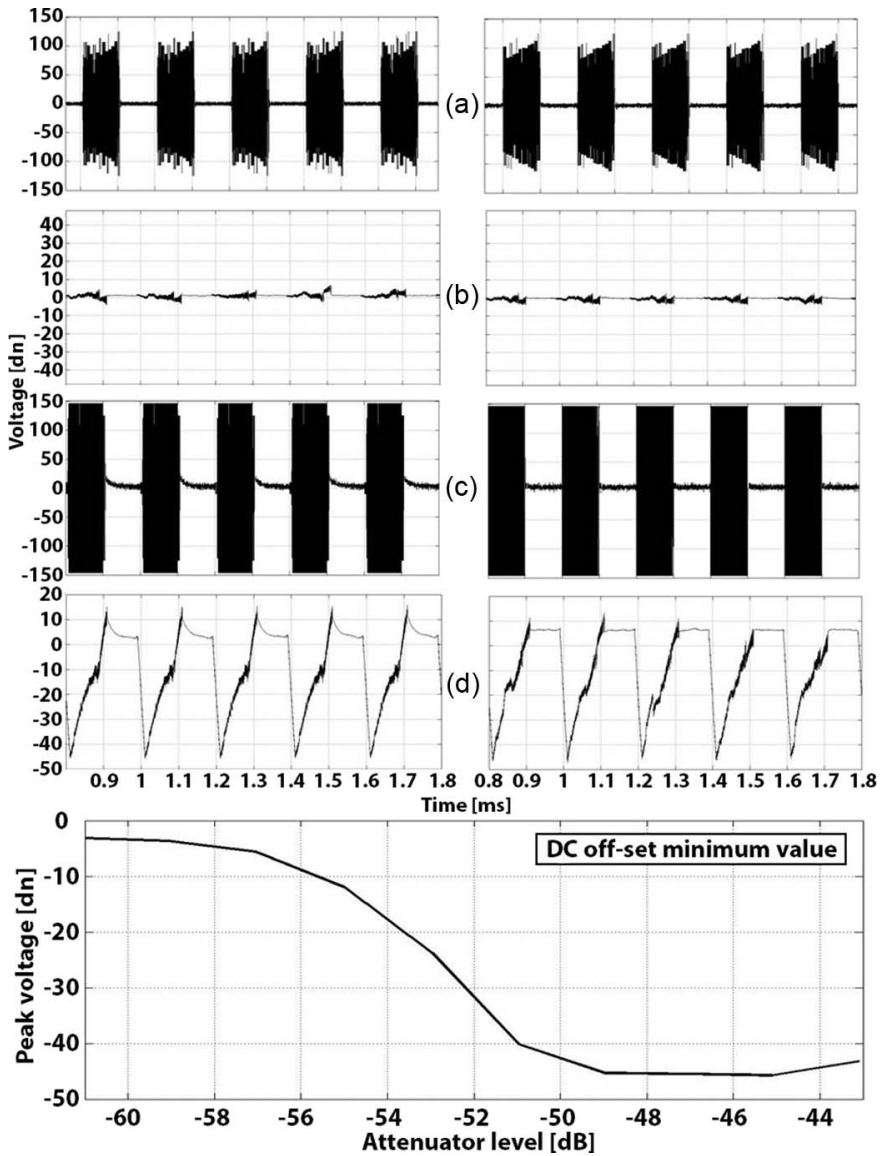

Fig. 3. [(a) left] DC offset observed on T56 engineering test and [(a) right] relative simulation. We show the result of applying the low-pass filter on a (d) saturated and an (b) unsaturated burst of the T56 test using a uniform moving window of $20 \mu \mathrm{s}$. The lower panel indicates the minimum value of dc offset for different value of attenuator level. Saturation occurs at a value of $-58 \mathrm{~dB}$.

(Ps/Pss), and seafloor vertical roughness $\left(\sigma_{\mathrm{h}}\right)$ using the simulations described earlier. Best fit parameters are chosen through the least squares minimization of the observed versus simulated waveforms, and parameter distributions are determined by the Monte Carlo approach with 1000 realizations per unique set of input parameters. For a given set of input parameters, each Monte Carlo realization provides an independent simulation of the subsurface shape, leading to unique waveforms with distinct speckle and instrument noise characteristics. In the second step, we adopt an inversion model to derive information about the dielectric properties of the sea (e.g., liquid loss tangent) from the best fit parameters determined in step 1. The inversion model links the estimated intensity ratio and depths to the loss tangent of the liquid using a two-layer scenario and assuming a uniform surface and seafloor (i.e., same dielectric properties). Fig. 4 shows a block diagram of the inversion process.

We use this approach in order to retrieve information from each of the 35 Cassini bursts acquired over Ligeia Mare. Concerning the Ontario Lacus data analysis, we only show here the ability to retrieve depth and intensity information from saturated data. We show that simulated waveforms are able to well reproduce the effects of saturations, including pre- and postcursors. The complete analysis of this data set to obtain depth and composition of Ontario Lacus will be discussed in a separate work.

\section{A. Ontario Lacus Saturated Data Simulation}

Applying the on-ground processing and superresolution techniques to the T49 altimeter data acquired over Ontario Lacus reveals typical double-peaked waveforms indicative of surface and subsurface detections. The waveforms, however, also show distortion caused by a severe saturation at the receiver. Fortunately, the simulator described earlier can adequately represent saturated data products. Simulated waveforms reproduce artifacts caused by saturation, which appear as a temporally predictable precursor signal that is located at the same travel time with respect to the surface/subsurface reflection. Examples of such artifacts are found in terrestrial radar altimeter applications as explained in [21]. As shown in Fig. 5, the simulations are able to reproduce signal precursors caused by the high saturation level of the Ontario data.

\section{B. Bathymetry of Ligeia Mare}

By means of a Monte Carlo method along with the waveform fitting technique described earlier, we compare the received echoes of Ligeia Mare with a set of simulated waveforms from different model parameters. The model space is explored by taking into account the a priori knowledge of the maximum depth and intensity ratio from the previous work of Mastrogiuseppe et al. [10]. This a priori information is thus expressed by bounding the possible parameters of the model space as indicated in the following.

1) Depths: We chose a range of depths from 0 to $190 \mathrm{~m}$ with a $2-m$ step, and the index refraction of the media is assumed to be 1.32 as in [10].

2) Intensity ratio (Ps/Pss): The surface/subsurface reflection intensity ratio Ps/Pss is allowed to vary from 15 to $50 \mathrm{~dB}$ with a $1-\mathrm{dB}$ step. The upper limit of this interval is consistent with the noise floor level, while the lower limit is chosen from T91 observation and the step according to the expected radiometric resolution of a single burst.

3) Vertical roughness: The rms height variation of the roughness is set to vary from $0 \mathrm{~m}$ to depth/4 at a 2-m step. Values of roughness greater then depth/4 are not expected as the spread of subsurface echoes always indicated an rms roughness smaller than depth/4 at Ligeia Mare [10].

In order to reduce the computational time and simplify the inversion process, we generated a lookup table where we collected all different Monte Carlo realizations of the model space. The lookup table consists of a multidimensional matrix storing 1000 different Monte Carlo realizations for each of the 30000 possible combinations of the model parameters, resulting in 30 million simulated waveforms.

Each realization of the 30000 element lookup table is compared to the observed Cassini bursts in order to estimate the best fit model parameters and generate posterior probability density functions of the parameters of interest ( i.e., depth, intensity ratio (Ps/Pss), and subsurface rms vertical height). Each of the simulated and real echoes are subject to incoherent averaging and superresolution processing. The resulting waveform is windowed $(2 \mu \mathrm{s})$ and normalized around its first surface peak reflection, and the resulting time vector containing the 


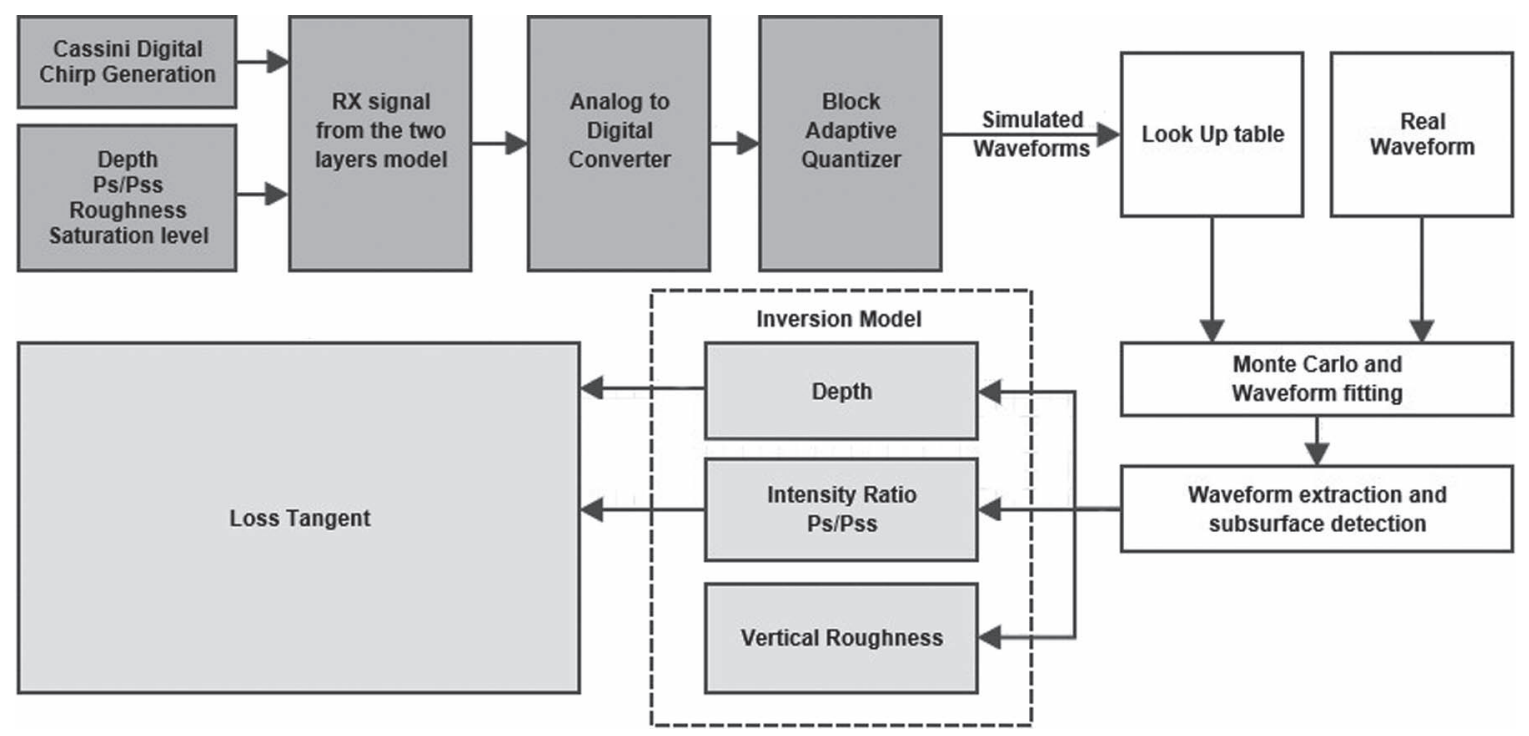

Fig. 4. Block diagram of the inversion processing.

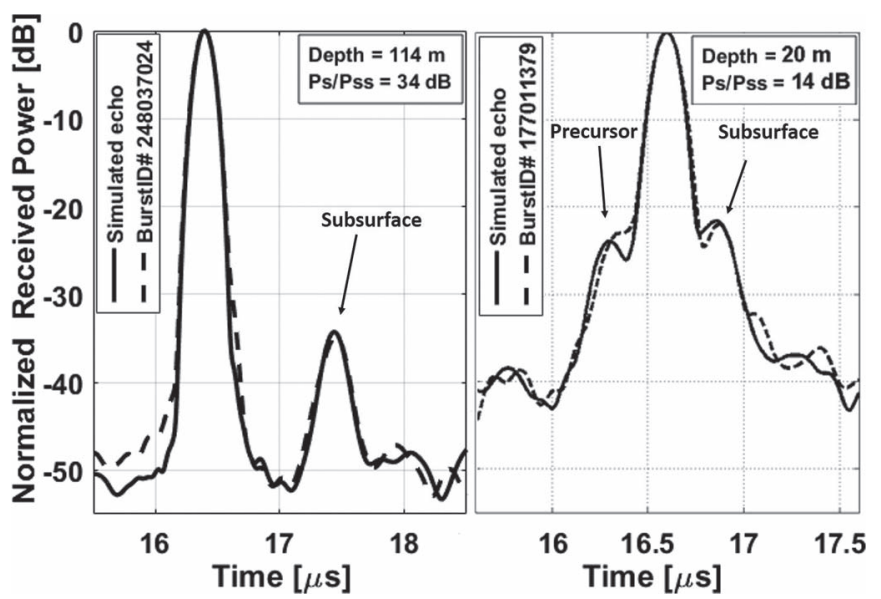

Fig. 5. (Left panel) Example of real waveform and synthetic waveform for T91 observation of Ligeia. (Right panel) Example of real and simulated waveforms acquired on T49 on Ontario Lacus. This last example shows how the simulated waveform is able to reproduce the artifact that appears as a precursor with respect to the surface reflection.

signal of interest (i.e., surface and subsurface reflection) is fitted to the simulated waveforms equally processed. The estimation is performed via a least squares procedure, minimizing a 3-D (Ps/Pss, depth, and surface roughness) error function.

In order to generate the estimates and relative errors, we calculate the maximum a posteriori probability (MAP) and the interval of confidence for each parameter and for each observed waveform. An example of probability density function of the three parameters of interest is shown in Fig. 6. In Fig. 7, we show a comparison between the simulated and the observed T91 Ligeia Mare radargram and an individual waveform.

\section{Bathymetry: Discussion and Results}

The obtained radargram using the Monte Carlo approach and waveform fitting is consistent with the results shown in [10] (see Fig. 7). However, the method adopted here permits to quantify an error for each individual radar observation. In Fig. 8, we

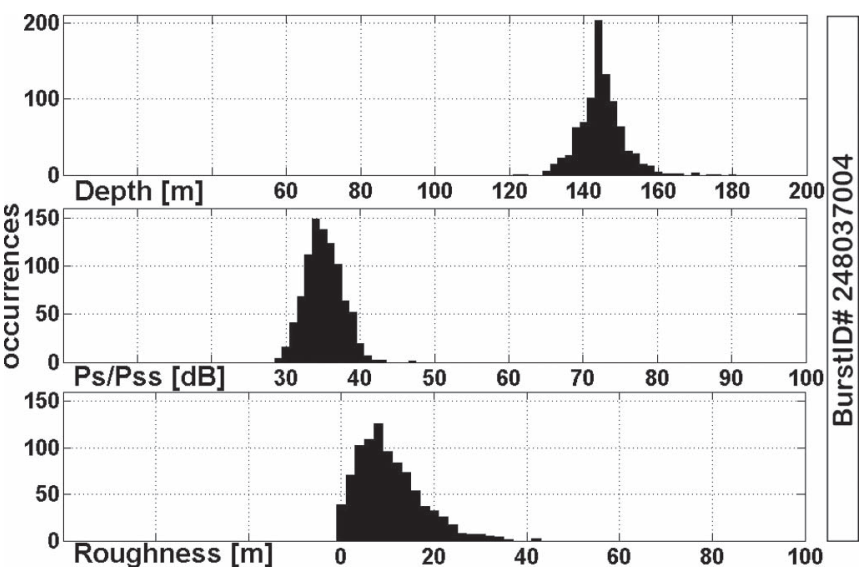

Fig. 6. Example of a posterior distribution of depth, intensity ratio, and vertical roughness for burst ID \#30.

show the bathymetry obtained from the proposed technique, errors on depth measurements range from about 5 to $50 \mathrm{~m}$, mostly depending on the SNR of the subsurface echoes and roughness. Large errors at the central portion of Ligeia are given by the low SNR due to the large attenuation of the deepest part of the sea.

The intensity ratio in Fig. 8(b) is also consistent with the result presented in the supplementary info in [10].

\section{LOSS TANGENT ESTIMATION}

In order to represent the backscattering of the returned waveforms, an appropriate model that describes the echo received from liquid bodies is needed. The amplitude ratio of the two main reflections (i.e., surface and subsurface) is mainly governed by the depth, dielectric properties of the liquid/seafloor, and the roughness at the interfaces.

We represent the backscatter received at the air/liquid and liquid/bottom interfaces using a two layer model similar to the 


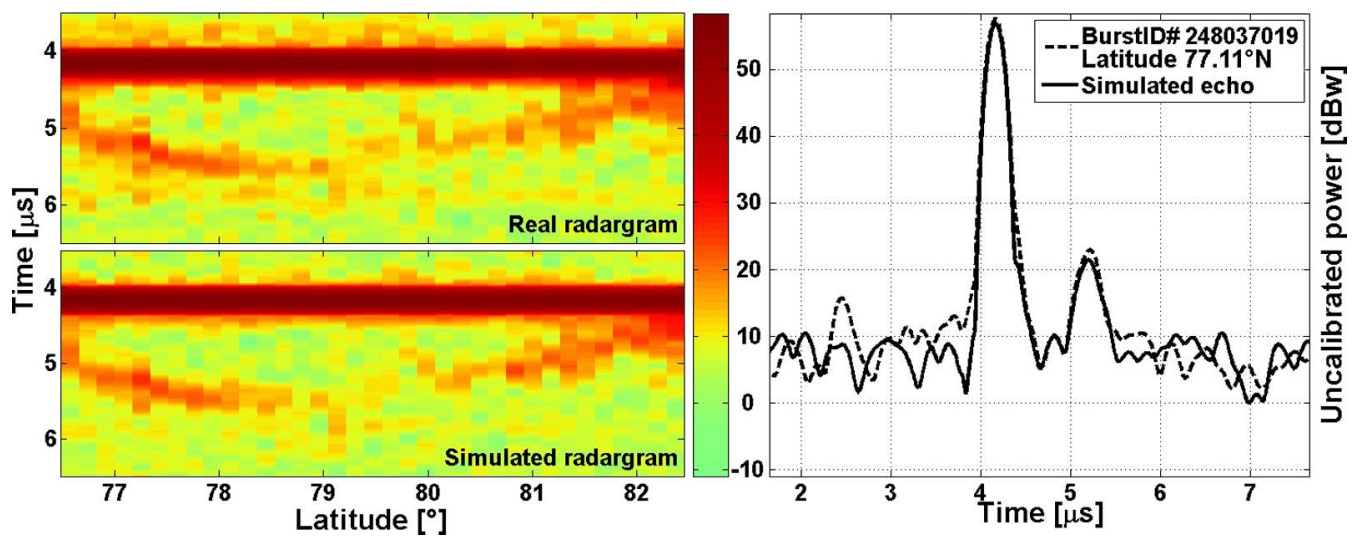

Fig. 7. (a) Comparison of (upper panel) the observed and (lower panel) the simulated radargram of T91 observation of Ligeia Mare. (b) Comparison of (dashed line) real observed data and (thick line) synthetic waveform calculated for the best fit model. Simulated and real waveforms include the first specular reflection and the secondary peak from the seafloor.

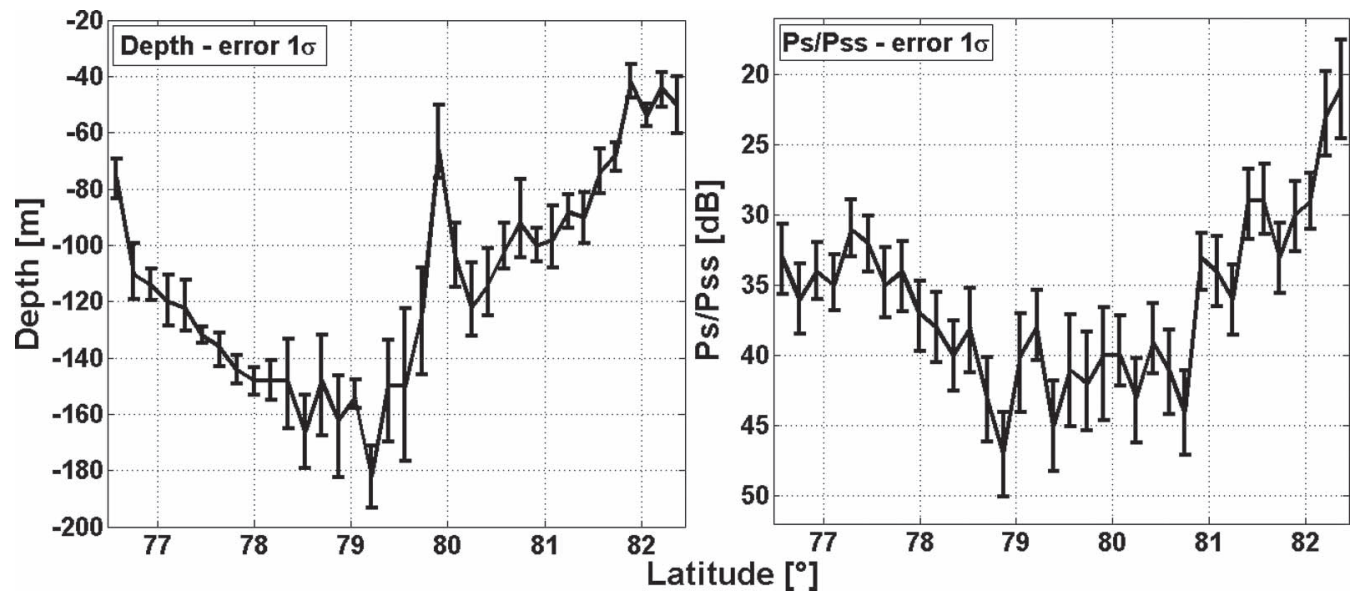

Fig. 8. Waveform fitting results. (Left) Bathymetry of Ligeia and relative errors. (Right) Intensity ratio Ps/Pss of Ligeia and relative errors.

ones presented in [29], where the backscattering at the surface and that at the subsurface are defined as

$$
\sigma_{s}=\Gamma_{s} * f_{s}, \quad \sigma_{s s}=\Gamma_{s s} * f_{s s} .
$$

The received intensity ratio Ps/Pss is related to the backscattering ratio $\sigma_{s} / \sigma_{s s}$ by the following formula (in decibel scale):

$$
\left.\frac{P_{s}}{P_{s s}}\right|_{d B}=\left.\Gamma_{s}\right|_{d B}-\left.\left(1-\Gamma_{s}\right)^{2}\right|_{d B}-\left.R_{12}\right|_{d B}+\left.K\right|_{d B}+\left.\frac{f_{s}}{f_{s s}}\right|_{d B} .
$$

The terms $f_{s}$ and $f_{s s}$ are the scattering terms which take into account the losses due to the roughness at the interfaces of the surface liquid and subsurface seafloor, respectively, $\Gamma_{\mathrm{s}}$ is the surface Fresnel reflectivity and $\Gamma_{\mathrm{s}}$ is the subsurface reflectivity (calculated at depth d), $\mathrm{K}$ (decibel scale) is the liquid attenuation (at delay $\Delta \tau$ ), and $\mathrm{R}_{12}$ is the Fresnel reflection coefficient. The Fresnel reflection coefficients and liquid attenuation are given by

$$
\begin{aligned}
\left.\Gamma_{s}\right|_{d B} & =10 \log 10\left|\frac{1-\sqrt{\varepsilon_{r 1}}}{1+\sqrt{\varepsilon_{r 1}}}\right|^{2} \\
\left.R_{12}\right|_{d B} & =10 \log 10\left|\frac{\sqrt{\varepsilon_{r 1}}-\sqrt{\varepsilon_{r 2}}}{\sqrt{\varepsilon_{r 1}}+\sqrt{\varepsilon_{r 2}}}\right|^{2} \\
K & =27 * \tan \Delta * f * \Delta \tau
\end{aligned}
$$

where $\varepsilon_{\mathrm{r} 1}$ and $\varepsilon_{\mathrm{r} 2}$ are the dielectric properties of the liquid and seafloor, respectively, $\tan \Delta$ is the loss tangent of the liquid, $\mathrm{f}$ is the operative frequency (in megahertz) of the radar, and $\Delta \tau$ (in microseconds) is the two-way travel time distance between the first and the second reflection.

Assuming uniformity for the surface and for the subsurface (i.e., same dielectric properties and scattering losses along the region of interest), we can determine the loss tangent of liquid using the following formulas:

$$
\frac{P_{s}}{P_{s s}}(\Delta \tau)=A+B * \Delta \tau
$$

where $\mathrm{A}$ is related to the dielectric properties of liquid and the angular coefficient B is the specific attenuation (in decibels per microsecond) of the liquid and directly related to the loss tangent via

$$
\tan \Delta=\frac{B}{27} / f .
$$

Parameters A and B are estimated via the linear regression of the amplitude ratio Ps/Pss as a function of depth. 

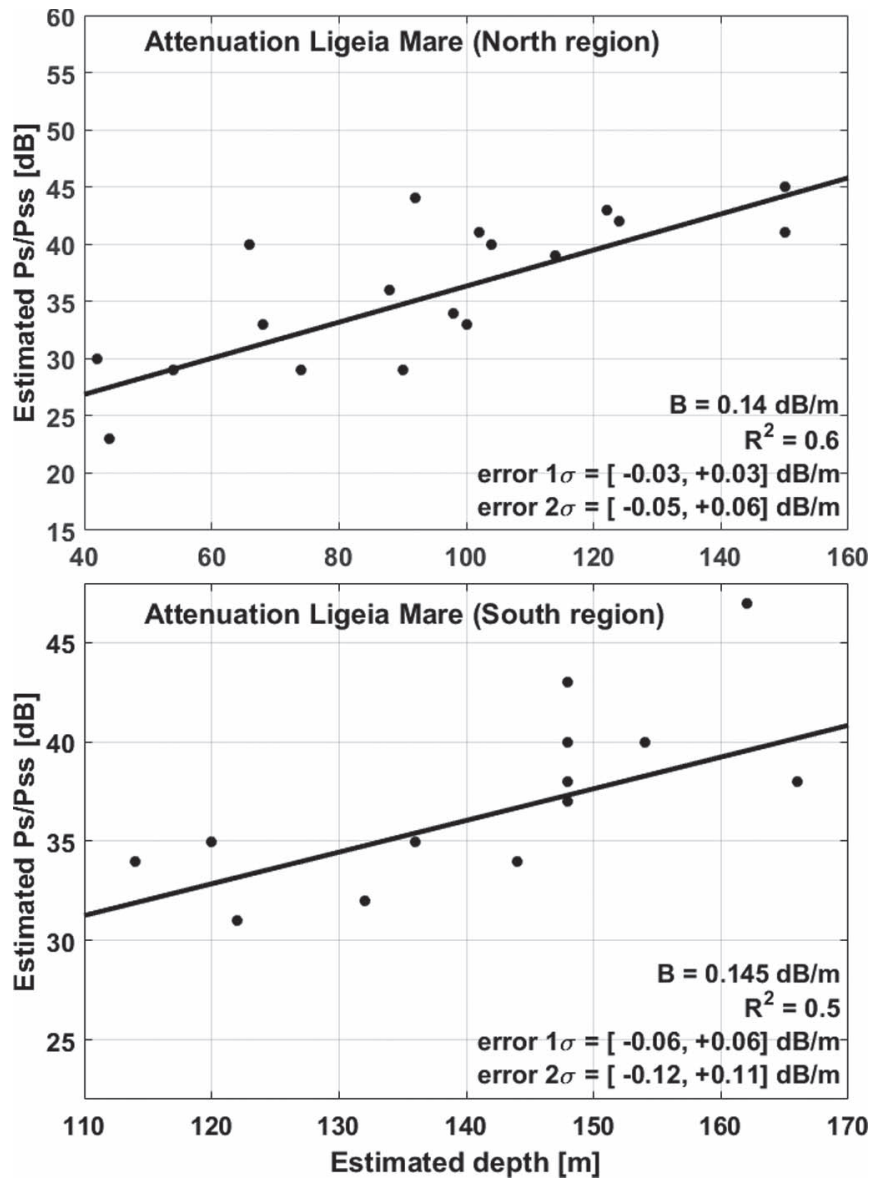

Fig. 9. Upper and bottom panels indicate a linear regression of amplitude ratio Ps/Pss versus depths of the $\mathrm{N}$ region and $\mathrm{S}$ region.

While $\mathrm{B}$ represents the specific attenuation of the liquid obtained from the angular coefficient of the regression, the parameter A takes into account the surface and subsurface reflectivities and is the y-intercept. Fig. 9 shows a linear regression obtained from two different data sets that represent the North and South regions of Ligeia Mare as explained in the following paragraph.

\section{A. Ligeia Liquid Loss Tangent: Results and Discussion}

We found that the subsurface amplitude received power has a trend that follows the estimated bathymetry (see Fig. 8). We attribute this behavior to be caused by the attenuation of the signal as a function of depth.

Best fit model parameters across Ligeia appear to be separated into two distinct sets. In general, the bursts acquired at the southern region of Ligeia ("S") have a very narrow distribution of depths (see an example in Fig. 6), low roughness, and values of Ps/Pss lower than those found in the northern region ("N"). One possible explanation of this behavior is that the overall seafloor of Ligeia is not completely uniform (i.e., different roughness or permittivity at the subsurface) and the $S$ region could have a smoother and more compact subsurface than the $\mathrm{N}$ region.

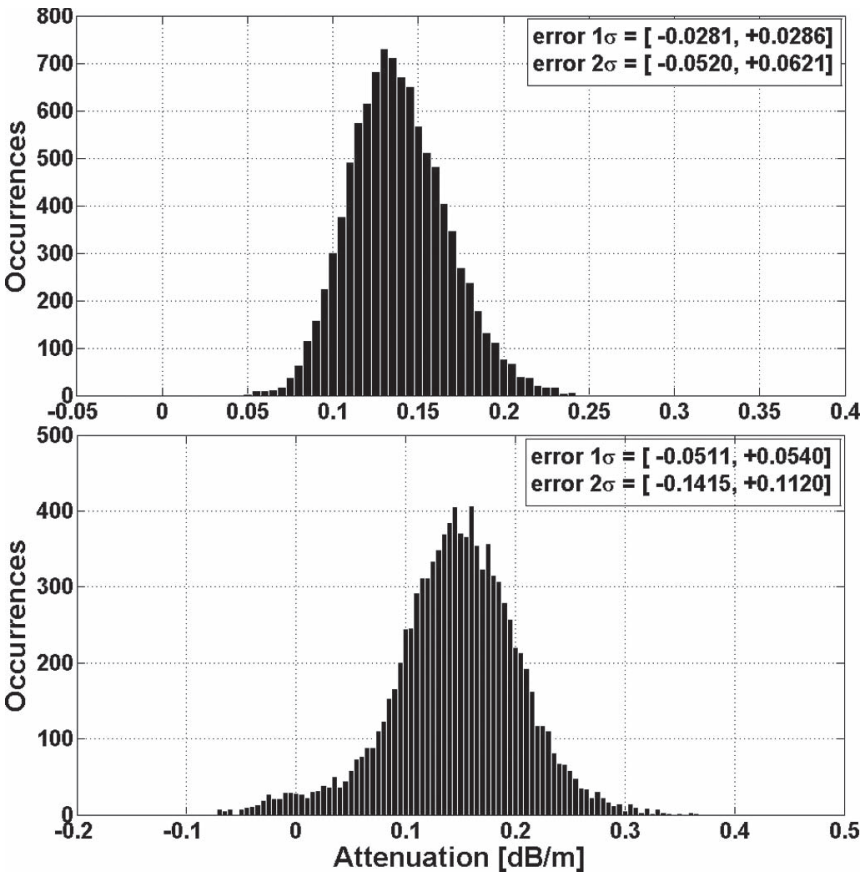

Fig. 10. Specific attenuation distribution for the (upper panel) North region and (lower panel) South region.

Alternatively, the surface backscatter could be nonuniform.

We apply this analysis to the two different regions $\mathrm{S}$ and $\mathrm{N}$, which are composed of 18 and 13 bursts, respectively. We do not consider for our analysis the two bursts acquired from $79.5^{\circ} \mathrm{N}$ to $80^{\circ} \mathrm{N}$ in latitude where the two regions appear divided, and we do not use two bursts acquired close to the shoreline. In order to evaluate the MAP and relative errors of attenuation distribution, for each individual data set (i.e., the $\mathrm{S}$ and $\mathrm{N}$ regions), we generated 10000 possible linear regressions obtained by randomly selecting a different value of depth and Ps/Pss from each individual a posteriori distribution obtained at the first step of the inversion processing. Inverting the two subsets independently, we obtained an estimation of the $\mathrm{B}$ parameter (specific attenuation) equal to $16.1 \mathrm{~dB} / \mu \mathrm{s} \pm 3.5 \mathrm{~dB} / \mu \mathrm{s}$ (or the correspondent $0.14 \mathrm{~dB} / \mathrm{m} \pm$ $0.03 \mathrm{~dB} / \mathrm{m}$ assuming the real part of the dielectric constant equal to 1.7) for the $\mathrm{N}$ zone and $16.7 \mathrm{~dB} / \mu \mathrm{s} \pm 5.7 \mathrm{~dB} / \mu \mathrm{s}$ (or the correspondent $0.145 \mathrm{~dB} / \mathrm{m} \pm 0.05 \mathrm{~dB} / \mathrm{m}$ ) for the $\mathrm{S}$ zone (see Fig. 10). These values result in loss tangents of $4.3 \times 10-5 \pm 1.2 \times 10-5$ and $4.5 \times 10-5 \pm 1.5 \times 10-5$ for the $\mathrm{N}$ and $\mathrm{S}$ regions, respectively. Assuming that the liquid composition is the same for both regions and that the analyses and observations are independent, the average loss tangent is $4.4 \times 10^{5} \pm 0.9 \times 10^{-5}$. All of the errors reported herein represent the $68 \%$ confidence interval (one-sigma).

The inversion analysis described herein provides an update on the loss tangent of Ligeia Mare from the previous estimate of $3 \times 10^{-5} \pm 1 \times 10^{-5}$ by Mastrogiuseppe et al. [10] to $4.4 \times 10^{-5} \pm 0.9 \times 10^{-5}$. Although the one-sigma errors of the previous and new estimates overlap, the average loss tangent is appreciably higher than that in [10]. We attribute this difference to systematic errors introduced by the assumptions made by Mastrogiuseppe et al. [10] as well as mixing data from 
the $\mathrm{N}$ and $\mathrm{S}$ regions in the original analysis. For example, the more rigorous model presented herein accurately reproduces the effects of noise and speckle, permitting more robust estimations of Ps/Pss. Mastrogiuseppe et al. [10] assumed that noise did not affect the measurement of Ps/Pss, which may have systematically biased larger values of Ps/Pss when the magnitude of the subsurface return (Pss) approached the noise level. Furthermore, the analysis of Mastrogiuseppe $e t$ al. [10] combined data from the $\mathrm{S}$ and $\mathrm{N}$ regions of Ligeia Mare, which this paper shows have difference subsurface scattering characteristics.

Within a given subregion, linear regression can also be used to estimate the A parameter, which takes into account both surface and subsurface dielectric properties as well as roughness. The result indicates that the backscattering ratio of area $\mathrm{N}$ is lower than that of area $\mathrm{S}$ (see Fig. 9). Distributions obtained from the bursts acquired in the northern area are also indicative of a larger vertical roughness at the radar footprint scale. This is also confirmed by the SAR imaging that shows a more complex subsurface scenario at the 300-m pixel scale, which could cause the observed roughness. We note that the $\mathrm{N}$ area includes the terminus of a large fluvial system that may be depositing material in Ligeia's seabed and contributing to the observed roughness.

\section{B. Liquid Composition}

We assume that the liquid in Ligeia Mare is a ternary mixture of methane, ethane, and nitrogen, based on the following.

1) Ethane and methane are the most abundant materials on Titan that have loss tangents low enough to match our observed value. They are liquid under Titan conditions (no solid compounds have the requisite low loss tangent).

2) The nitrogen is a dissolved component whose mole fraction in the liquid is determined by the partial pressure (1.5 bars) of nitrogen in contact with the open seas, and that nitrogen solubility is determined from measurements of nitrogen measured in both pure ethane/methane [38] as well as mixtures [44].

3) The microwave absorption coefficient of the mixture at $2.2-\mathrm{cm}$ wavelength is determined from a combination of its individual components using the Lorentz-Lorenz mixing rule [40].

We take the uncertainties in the pure component loss tangents (the "nominal nonconservative" values) from [39] and use the Lorenz-Lorentz relation [40] to determine the dielectric properties of the mixture. Given these assumptions, the measured loss tangent for Ligeia yields a best fit composition (volume fraction) of $71 \% \mathrm{CH}_{4}, 12 \% \mathrm{C}_{2} \mathrm{H}_{6}$, and $17 \% \mathrm{~N}_{2}$. Such values are generally consistent with the equilibrium models of Titan's sea composition, such as the one proposed in [41] and the hypothesis in [42] where latitudinal variability in precipitation frequency may enhance the methane fraction of Ligeia Mare as compared to the more southern Kraken Mare.

\section{CONCLUSION}

In this paper, we describe a method based on a Monte Carlo approach and waveform fitting to retrieve bathymetry and composition from the altimetric observation of Titan's liquid bodies. We demonstrate how to extract information from the saturated data of Ontario Lacus, and we provide a new analysis to update the Ku-band attenuation of Ligeia Mare that results in a new more robust estimate for its loss tangent and composition. The technique presented herein is general and can be adapted to retrieve best fit model parameters from radar sounding data sets.

\section{ACKNOWLEDGMENT}

The authors would like to thank the Italian Space Agency (ASI) for providing the CPAD SW Tool developed by CORISTA to process the Cassini radar data in altimeter mode.

\section{REFERENCES}

[1] G. Picardi et al., "MARSIS: Mars advanced radar for subsurface and ionosphere sounding," in Proc. Mars Express: Sci. Payload, ESA Pub. Div., 2004, pp. 51-69.

[2] R. Seu et al., "SHARAD sounding radar on the Mars reconnaissance orbiter," J. Geophys. Res., vol. 112, May 2007, Art. no. E05S05.

[3] T. Ono et al., "Instrumentation and observation target of the Lunar Radar Sounder (LRS) experiment on-board the SELENE spacecraft," Earth, Planets Space, vol. 60, no. 4, pp. 321-332, Apr. 2007.

[4] M. E. Peters, D. D. Blankenship, and D. L. Morse, "Analysis techniques for coherent airborne radar sounding: Application to West Antarctic ice streams," J. Geophys. Res., vol. 110, no. B6, Jun. 2005, Art. no. B06303.

[5] D. D. Blankenship, D. A. Young, W. B. Moore, and J. C. Moore, "Radar sounding of Europa's subsurface properties and processes: The view from Earth," in Europa, R. T. Pappalardo, W. B. McKinnon, and K. K. Khurana, Eds. Tucson, AZ, USA: Univ. Arizona Press, 2009, pp. 631-654.

[6] L. Bruzzone et al., RIME: Radar for icy moon exploration, in Proc. IEEE IGARSS, Jul. 2013, pp. 3907-3910, doi: 10.1109/IGARSS.2013.6723686.

[7] Aharonson et al., Titan's Surface Geology. I. Mueller-Wodarg, C. Griffith, E. Lellouch, and T. Cravens, Eds. Cambridge, MA, USA: Cambridge Univ. Press, 2014, pp. 43-75.

[8] G. Picardi et al., "RADAR geomorphologic characterization of Titan," in Proc. Int. Conf. Radar, Brighton, U.K., 1992, pp. 254-257.

[9] W. R. Thompson and S. W. Squyres, "Titan and other icy satellitesDielectric properties of constituent materials and implications for radar sounding," Icarus, vol. 86, no. 2, pp. 336-354, Aug. 1990.

[10] M. Mastrogiuseppe et al., "The bathymetry of a Titan Sea," Geophys. Res. Lett., vol. 41, no. 5, pp. 1432-1437, Mar. 2014.

[11] R. D. Lorenz et al., "Titan's inventory of organic surface material," Geophys. Res. Lett., vol. 35, no. 2, pp. 2616-2628, Jan. 2008.

[12] G. Picardi et al., "MARSIS data inversion approach," in Proc. IEEE 4th Int. Workshop Adv. Ground Penetrating Radar, Napoli, Italy, 2007, pp. 256-260.

[13] K. Matsuoka, "Estimating englacial radar attenuation using depth profiles of the returned power, central West Antarctica," J. Geophys. Res., vol. 115, no. F2, Jun. 2010, Art. no. F0212.

[14] Z. Zhang, "Dielectric properties of the Martian south polar layered deposits: MARSIS data inversion using Bayesian inference and genetic algorithm," J. Geophys. Res., vol. 113, no. E5, May 2008. [Online]. Available: http://dx.doi.org/10.1029/2007JE002941

[15] C. Grima, "North polar deposits of Mars: Extreme purity of the water ice," Geophys. Res. Lett., vol. 36, no. 3, pp. 2-5, Feb. 2009, [Online]. Available: http://dx.doi.org/10.1029/2008GL036326

[16] J. W. Holt, "Radar sounding evidence for buried glaciers in the Southern Mid-Latitudes of Mars," Science, vol. 322, no. 5905, pp. 1235-1238, Nov. 2008. [Online]. Available: http://www.sciencemag.org/content/322/ $5905 / 1235$ 
[17] K. Matsuoka, D. Morse, and C. F. Raymond, "Estimating englacial radar attenuation using depth profiles of the returned power, central West Antarctica," J. Geophys. Res., vol. 115, no. F2, Jun. 2010, Art. no. F0212. [Online]. Available: http://dx.doi.org/10.1029/2009JF001496

[18] H. Zebker, "Surface of Ligeia Mare, Titan, from Cassini altimeter and radiometer analysis," Geophys. Res. Lett., vol. 41, no. 2, pp. 308-313, Jan. 2014.

[19] Wye et al., "Smoothness of Titan's Ontario Lacus: Constraint from Cassini RADAR specular reflection," Geophys. Res. Lett., vol. 36, Aug. 2009, Art. no. L16201.

[20] R. Kwok and W. T. K. Johnson, "Block adaptive quantization of Magellan SAR data," IEEE Trans. Geosci. Remote Sens., vol. 27, no. 4, pp. 375-383, Jul. 1989

[21] D. J. Wingham and C. G. Rapley, "Saturation effects in the Seasat altimeter receiver," Int. J. Remote Sens., vol. 8, no. 8, pp. 1163-1173, May 2007.

[22] A. Tarantola, Inverse Problem Theory and Methods for Model Parameter Estimation. Philadelphia, PA, USA: SIAM, Univ. City Sci. Center, 2005.

[23] E. R. Stofan, "The lakes of Titan," Nature, vol. 445, pp. 61-64, Jan. 2007.

[24] A. G. Hayes, "Hydrocarbon lakes on Titan: Distribution and interaction with a porous regolith," Geophys. Res. Lett., vol. 35, May 2008, Art. no. L09204.

[25] A. G. Hayes, "Transient surface liquid in Titan's polar regions from Cassini," Icarus, vol. 211, no. 1, pp. 655-671, Jan. 2011.

[26] A. G. Hayes, "Bathymetry and absorptivity of Titan's Ontario Lacus," J. Geophys. Res., vol. 115, Sep. 2010, Art. no. E09009.

[27] C. Elachi, "Radar: The Cassini Titan Radar Mapper," Space Sci. Rev., vol. 115, no. 1, pp. 71-110, Nov. 2004.

[28] R. K. Raney, "The delay/Doppler radar altimeter," IEEE Trans. Geosci. Remote Sens., vol. 36, no. 5, pp. 1578-1588, Sep. 1998.

[29] G. Picardi et al., "The radar system for the exploration of Titan," Il Nuovo Cimento, vol. 15, no. 6, pp. 1149-1161, Nov. 1992.

[30] G. Cascioli and R. Seu, "Surface backscattering evaluation by means of the facet model for remote sensing applications," Acta Astronautica, vol. 40, no. 11, pp. 849-857, Jun. 1996.

[31] G. Bernardi, "Innovative fractal-based models for radar altimeter data," Ph.D. dissertation, Dept. Elect. Eng., Federico II Univ., Naples, Italy, 2011.

[32] M. Mastrogiuseppe et al., "Titan dune heights retrieval by using Cassini Radar Altimeter," Icarus, vol. 230, pp. 336-354, Feb. 2014.

[33] G. Franceschetti and D. Riccio, Scattering Natural Surface and Fractal. Burlington, MA, USA: Elsevier, 2006.

[34] T. Hagfors, "Backscattering from undulating surface with application to radar returns from the moon," J. Geophys. Res., vol. 69, pp. 3779-3784, Sep. 1964.

[35] A. K. Sultan-Salem et al., "Modeling quasi-specular scattering from the surface of Titan," J. Geophys. Res., vol. 112, no. E5, May 2007, Art. no. E05012.

[36] L. C. Wye, "Radar scattering from titan and Saturn's icy satellites using the Cassini spacecraft," Ph.D. dissertation, Dept. Elect. Eng., Stanford Univ., Stanford, CA, USA, 2011.

[37] G. Alberti et al., "The Processing of Altimetric Data (PAD) System for Cassini RADAR," Memorie della Societa Astronomica Italiana Suppl., vol. 11, p. 68, 2007.

[38] H. Cheung and D. I.-J. Wang, "Solubility of volatile gases in hydrocarbon solvents at cryogenic temperatures," Ind. Eng. Chem. Fundam., vol. 3, no. 4, pp. 355-361, Nov. 1964, doi: 10.1021/i160012a014.

[39] K. Mitchell et al., "Composition of Ligeia Mare, Titan, from cryogenic laboratory measurements and bathymetry," Geophys. Res. Lett., vol. 42, no. 5, Mar. 2015, Art. no. 2434.

[40] M. Born and E. Wolf, Principles of Optics: Electromagnetic Theory of Propagation, Interference and Diffraction of Light, 7 th ed. Cambridge, U.K.: Cambridge Univ. Press, 1999.

[41] Tan et al., "Titan's liquids: Exotic behavior and its implications on global fluid circulation," Icarus, vol. 250, pp. 64-75, Apr. 2015.

[42] Lorentz, "The flushing of Ligeia: Composition variation across Titan's seas in a simple hydrological model," Geophys. Res. Lett., vol. 41, no. 16, pp. 5764-5770, Aug. 2014.

[43] A. G. Hayes, "The lakes and seas on titan," in Proc. Annu. Rev. Earth Planetary Sci., vol. 44, pp. 57-83, May 2015.

[44] M. J. Malaska et al., "The dissolved nitrogen fluffiness of Titan's lakes," in Proc. 47th Lunar Planetary Sci. Conf., Mar. 2016, p. 1729.

[45] A. G. Hayes et al., "Wind driven capillary-gravity waves on Titan's lakes: Hard to detect or non-existent," Icarus, vol. 225, no. 1, pp. 403-412, Jul. 2013.

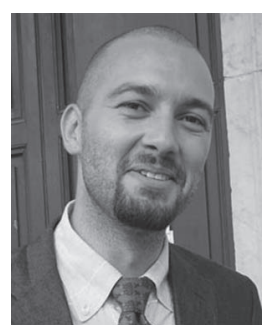

Marco Mastrogiuseppe was born in Atri (TE), Italy, in 1976. He received the B.S., M.S., and Ph.D. degrees in telecommunication engineering from the University of "Sapienza," Rome, Italy, in 2005 2008 , and 2012, respectively.

From 2012 to 2014, he was a Research Collaborator with the Dipartimento Elettronica e Telecomunicazione (DIET), La Sapienza University, Rome. Since 2014, he has been a Research Associate with the Cornell Center for Astrophysical Science, Cornell University, Ithaca, NY, USA. He is the author of several articles. His research interests include remote sensing, radar system, data processing, and sounder data analysis for planetary exploration. $\mathrm{He}$ received a Group Achievement Award from NASA in 2011, SHARAD Science Operation. He was nominated as Associate Team Member of Cassini RADAR Science Team in 2013. He is a team member of the SHARAD experiment on board MRO. Since 2015, he is a Coinvestigator of the NASA project: Radar for Europa Assessment and Sounding: Ocean to Near-surface.

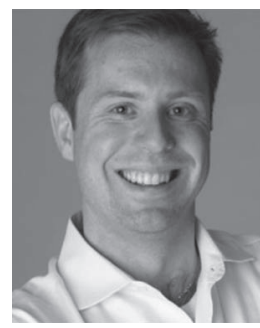

A. Hayes received B.S. degrees astronomy and astrobiology and the M.Eng. degree in applied physics from Cornell University, Ithaca, NY, USA, and the M.S. and Ph.D. degrees in planetary science from the California Institute of Technology, Pasadena, CA, USA.

$\mathrm{He}$ is an Assistant Professor of Astronomy at Cornell University and Director of the Spacecraft Planetary Imaging Facility. He uses spacecraft-based remote sensing to study the properties of planetary surfaces, interiors, and atmospheres. He is a Participating Scientist on the Cassini-Huygens Mission to Saturn, as well as a CoInvestigator on NASA's Europa Multiple Flyby Mission and the Mars2020 rover. He is a collaborator on the Mars Exploration Rover and Mars Science Laboratory.

Dr. Hayes is the winner of the Ronald Greeley Early Career Award of the Planetary Sciences Section of the American Geophysical Union and the Zeldovich Prize in Commission B of the Committee on Space Research . He is also a Kavli Fellow of the National Academy of Sciences and a NASA Early Career Fellow.

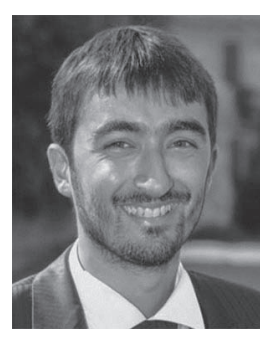

Interplanetary Society.
V. Poggiali was born in Genoa, Italy, in 1983. He received the B.S. and M.S. degrees in telecommunications engineering from Sapienza Università di Roma, Roma, Italy, in 2008 and 2011, respectively.

In the same university, he is a Research Assistant at the Department of Information, Electronics and Telecommunications and attending the Doctoral Program in Radar and Remote Sensing.

Mr. Poggiali is an Associate Member of the Cassini Radar Science Team, a member of the American Geophysical Union, and a Fellow of the British 


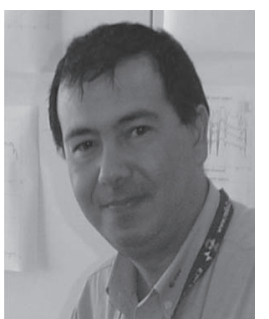

R. Seu was born in Carbonia (Cagliari) on February 18, 1959. He received the M.S. degree in electronics engineering and the Ph.D. degree from the "'Universita' degli Studi "La Sapienza" of Rome," Rome, Italy, in 1985 and 1990, respectively.

He has been an Assistant Professor since 1992 and is a Lecturer of the course of "Radar systems for space applications" and "Systems for air traffic control" at the "Universita' degli Studi "La Sapienza" of Rome. Since the doctoral degree, he has been working on radar systems and mainly on radars for planetary observations. Since 1993, he has been a member of the Cassini Radar Science Team, coinvestigator of the experiment CONSERT, payload of the ESA mission Rosetta and of the experiment MARSIS, radar sounder on board the ESA mission Mars Express. Since 2001, he is a Team Leader of the experiment SHARAD, a radar sounder payload of the NASA mission Mars Reconnaissance Orbiter.

Dr. Seu is a Referee of Planetary and Space Science, the IEEE TRANSACTIONS ON GEOSCIENCE AND REMOTE SENSING, the IEEE TRANSACTIONS on AEROSPACE AND ELECTRONIC Systems, and of the scientific journal Icarus, and he has been a member of the scientific committee of the IEEE Radar Conference 2008, held in Rome on May 2008. He has received the "Certificate of appreciation" from the JPL/MRO Project and the "AWARD" from NASA Headquarters for the results achieved by the SHARAD Team in the design and development phase of SHARAD. Last July 2008, he has received from the IEEE Geoscience and Remote Sensing Society the "2008 Interactive Session Prize Paper Award" for the paper "SHARAD design and operation" presented at the IGARSS 2007 conference.

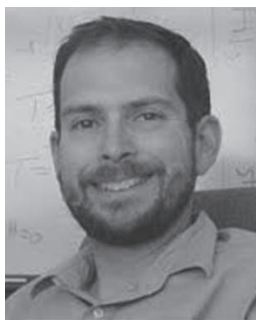

Jonathan I. Lunine received the B.S. degree in physics and astronomy from the University of Rochester, Rochester, NY, USA, and the M.S. and $\mathrm{Ph} . \mathrm{D}$. degrees in planetary science from the California Institute of Technology, Pasadena, CA, USA.

$\mathrm{He}$ is the Director of the Center for Radiophysics and Space Research and a David C. Duncan Professor in the Physical Sciences at Cornell University, Ithaca, NY, USA. He is interested in how planets form and evolve, what processes maintain and establish habitability, and what the limits of environments capable of sustaining life are. He is an interdisciplinary scientist on the Cassini Saturn Orbiter and Huygens Probe. He is a coinvestigator on the Juno mission launched in 2011 to Jupiter and an interdisciplinary scientist for the James Webb Space Telescope.

Dr. Lunine is the winner of the Harold C. Urey Prize of the DPS/American Astronomical Society, the Macelwane Medal of the American Geophysical Union (AGU), the Zeldovich Prize in Commission B of COSPAR, and the Basic Science Award of the International Academy of Astronautics. He is a Fellow of the AGU and the American Association for the Advancement of Science.

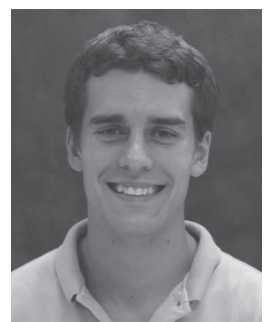

J. D. Hofgartner was born in Windsor, ON, Canada in 1988. He received the B.S. degree in physics, cooperative program, from the University of Waterloo, ON, Canada, in 2011 and the M.S. degree in astronomy from Cornell University, Ithaca, NY, USA, in 2014.

As an undergraduate, he was a Research Assistant at the Perimeter Institute for Theoretical Physics and SNOLAB and a Geophysical Data Processor at Sander Geophysics. He is currently with the Cornell Center for Astrophysical Science, Cornell University, Ithaca, NY, USA. He is an Associate Member of the Cassini Radar Team and the author of three articles.

Mr. Hofgartner is a member of the American Geophysical Union (AGU) and a recipient of a NASA Postdoctoral Fellowship, the Cornell University Cranson and Edna Shelley Graduate Research Award in 2015, the AGU Outstanding Student Paper Award in 2014, and the Natural Sciences and Engineering Research Council of Canada Master's and Doctoral Post Graduate Scholarships in 2011-2012 and 2012-2015. 